\title{
Reply to Comment by Lamy et al. on "Locating the source field lines of Jovian decametric radio emissions"
}

\author{
YuMing Wang ${ }^{1,2,3^{*}}$, RuoBing Zheng ${ }^{1,2}$, XianZhe Jia4, ChuanBing Wang ${ }^{1,2}$, Shui Wang ${ }^{1,2}$, and V. Krupar ${ }^{5,6,7}$ \\ ${ }^{1}$ CAS Key Laboratory of Geospace Environment, School of Earth and Space Sciences, University of Science and Technology of China, Hefei 230026, China; \\ ${ }^{2}$ CAS Center for Excellence in Comparative Planetology, Hefei 230026, China; \\ ${ }^{3}$ Mengcheng National Geophysical Observatory, School of Earth and Space Sciences, University of Science and Technology of China, Hefei 230026, China; \\ ${ }^{4}$ Department of Climate and Space Sciences and Engineering, University of Michigan, Ann Arbor, MI 48109-2143, USA; \\ 5 Universities Space Research Association, Columbia, Maryland, USA; \\ ${ }^{6}$ NASA Goddard Space Flight Center, Greenbelt, Maryland, USA; \\ ${ }^{7}$ Department of Space Physics, Institute of Atmospheric Physics, The Czech Academy of Sciences, Prague, Czech Republic
}

Citation: Wang, Y. M., Zheng, R. B., Jia, X. Z., Wang, C. B., Wang, S., and Krupar, V. (2022). Reply to Comment by Lamy et al. on "Locating the source field lines of Jovian decametric radio emissions". Earth Planet. Phys., 6(1), 13-17. http://doi.org/10.26464/epp2022019

\begin{abstract}
Locating the source of decametric (DAM) radio emissions is a key step in the use of remote radio observations to understand the Jovian magnetospheric dynamics and their interaction with the planet's moons. Wang YM et al. (2020) presented a method by which recorded arc-shaped DAM emissions in the radio dynamic spectra can be used to locate the source of a DAM. An lo-related DAM event on March 14, 2014 was used to demonstrate the method. A key parameter in the method is whether the DAM is emitted in the northern or the southern hemisphere; the hemisphere of origin can be determined definitively from the polarization of the emission. Unfortunately, polarization information for the emission on March 14, 2014 event was not recorded. Our analysis assumed the source to be in the northern hemisphere. Lamy et al. (2022) argue convincingly that the source was probably in the southern hemisphere. We appreciate the helpful contribution of Lamy et al. (2022) to this discussion and have updated our analysis, this time assuming that the DAM source was in the southern hemisphere. We also explore the sensitivity of our method to another parameter - the height at which the value of $f_{\mathrm{ce}, \max }$ which is the maximal electron cyclotron frequency reached along the active magnetic flux tube, is adopted. Finally, we introduce our recent statistical study of 68 DAM events, which lays a more solid basis for testing the reliability of our method, which we continue to suggest is a promising tool by which remote radio observations can be used to locate the emission source of Jovian DAMs.
\end{abstract}

Keywords: radio decametric emissions; Jovian magnetosphere; energetic electrons

\section{Updated Results of the lo-DAM on 2014 March 14}

A detailed description of our method can be found in Wang YM et al. (2020) (hereafter W20). The method's core formula to constrain the source of a DAM is given in Hess et al. (2008):

$$
a=\arccos \left[\frac{v}{c}\left(1-\frac{f_{\mathrm{ce}}}{f_{\mathrm{ce}, \max }}\right)^{-1 / 2}\right],
$$

in which $a$ is the emission angle, $v$ is the speed of the energetic electrons, $c$ is the speed of light, $f_{\text {ce }}$ is the electron's cyclotron frequency, and $f_{\mathrm{ce}, \max }$ is the maximum cyclotron frequency that the electrons can reach along the active field line from which the DAM is emitted. This formula is based on the loss-cone distribution of electrons, which has been supported by Juno in situ observations (Louarn et al., 2017, 2018; Louis et al., 2020). Another con-

Correspondence to: Y. M. Wang, ymwang@ustc.edu.cn

Received 08 JAN 2022; Accepted 13 JAN 2022.

Accepted article online 13 JAN 2022.

(C) 2022 by Earth and Planetary Physics. straint in the method is the hemisphere from which the DAM emits. According to the arc shape of a DAM, we can determine whether it is from the planet's eastern or western hemisphere; from the DAM's polarization, we can determine whether it is from the northern or southern hemisphere. Lacking polarization information for the DAM on March 14, 2014, which was the case studied in W20, we assumed it to be from the northern hemisphere. Lamy et al. (2022) (hereafter L22) have presented evidence that the DAM was probably from the southern hemisphere. We have revisited the March 14, 2014, event accordingly, updating the hemisphere, and present our new results below.

Figure 1 displays the source location of the DAM, based on data from Wind and STEREO-A (ST-A) and STEREO-B (ST-B) satellites; this is the update of Fig. 5 of W20. Detailed results are shown in Figure 2. It is suggested that the footprints of the source field lines were located between south longitudes $220^{\circ}$ and $290^{\circ}$, and just ahead of the lo footprint. The rotational speed of the lo footprint in inertial coordinates is about $0.37 \Omega$, on average $(\Omega$, is the self-rotation speed of Jupiter); that of the footprint of the DAM source is 


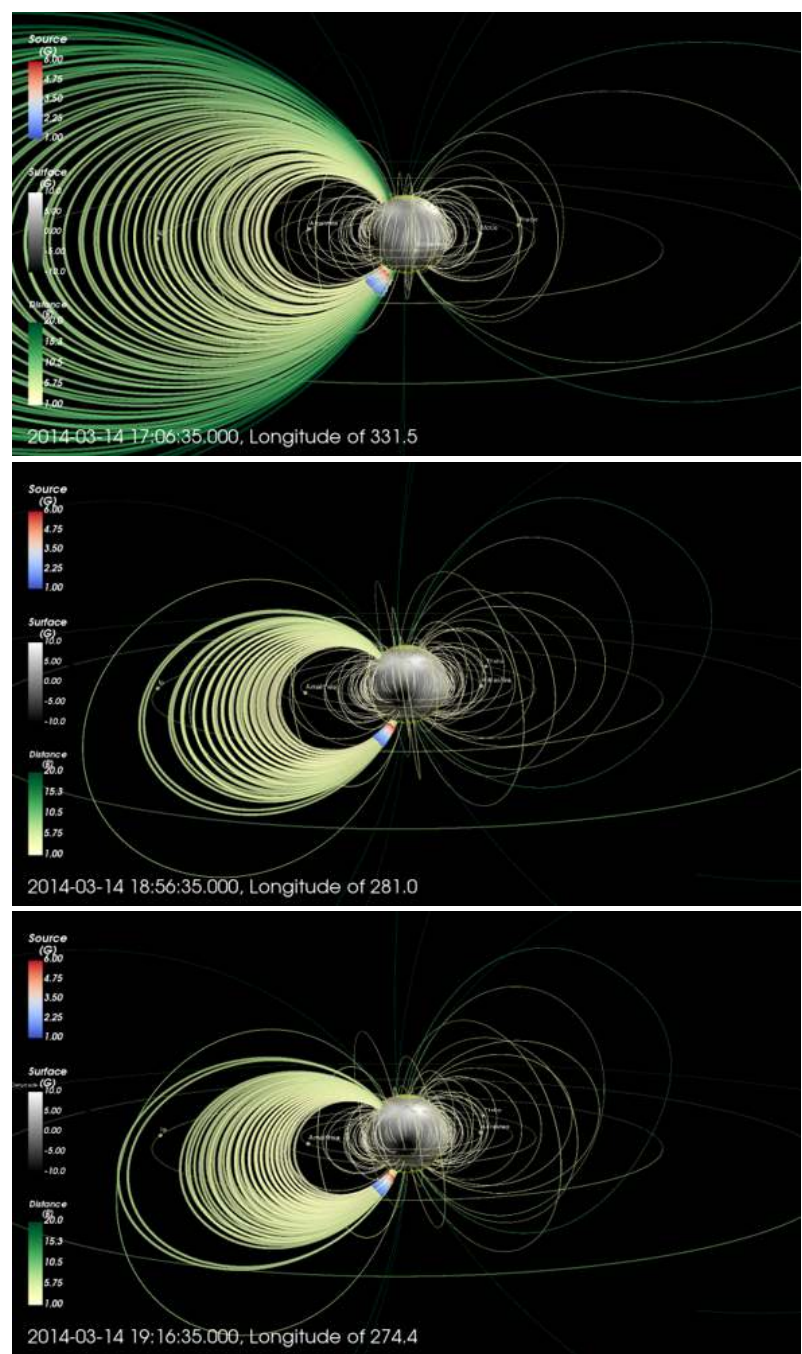

Figure 1. The three panels from up to down show the located DAM source based on the radio dynamic spectrum observed the Wind/WAVES, ST-A/WAVES and ST-B/WAVES satellites, respectively. The $1 / 15.4$ flattened surface of $B_{\mathrm{r}}$ at one $R_{\mathrm{J}}$ is indicated by the grayscaled ellipsoid at the center and is scaled by the gray bar on the left. Thin curves show the background magnetic field lines; colors, scaled by the green bar on the left, denote the distances of the tops of these field lines away from the center of Jupiter. All the selected field lines are thicker ones, and the located DAM source corresponding to the emission frequency from $5-16 \mathrm{MHz}$ is marked by color-coded dots along these field lines. The color of the DAM source indicates the local magnetic field strength, also according to the color scale on the left. Jupiter's major moons, including lo, Europa, Ganymede, Thebe, Amalthea, Adrastea, and Metis, as well as their orbits, are marked by small balls and nearly round curves. The time and longitude indicated at the lower-left corner of each panel give the time at Jupiter and the longitude of the center of Jupiter's disk, respectively.

about $0.38 \Omega$, tops of the active lines are also ahead of lo in longitude with apparent rotational speed of about $0.32 \Omega$, causing the lead to increase from about $15^{\circ}$ to more than $20^{\circ}$. The top distances to the active lines from Wind are mainly around $4-15 R_{\mathrm{J}}\left(R_{\mathrm{J}}\right.$ is the Jovian planetary radius) and $3-5 R$, as measured from ST-A and ST-B. The emission angles fall in the range of $55^{\circ}-80^{\circ}$ decreasing slightly with time. Accordingly, the electron energies increase with time from about $5 \mathrm{keV}$ to about $9 \mathrm{keV}$ on average. And as governed by Equation (1), the emission angle increases with the field line distance; the energy of the associated electrons decreases with the distance. Some key parameters have been summarized in Table 1. It could be found that notable differences in, e.g., apparent rotation speed, field line top distance, emission angle and electron energy, may occur when wrong source hemisphere is assumed, pointing out the importance of the predetermination of the source hemisphere of a DAM emission when applying our method.

\section{Influence of the Selection of $\boldsymbol{f}_{\mathrm{ce}, \max }$ on the Results}

In W20, one of the key parameters, $f_{\text {ce,max, }}$ was chosen to be the value measured at the surface of Jupiter (as an approximation to the top of the planet's ionosphere). In principle, since we are assuming the loss-cone distribution, we should choose the value of $f_{\text {ce,max }}$ to be the height at which the greatest number of associated energetic electrons are lost due to collisions. If an aurora is taking place, that height would be the height of the aurora. As pointed out by L22, the height of peak emission of the lo UV footprint ranges from about 500 to $1300 \mathrm{~km}$ with mean height of about 900 km (Bonfond et al., 2009).

To evaluate how the height assumption influences the results of our model, we have re-run the procedure with the value of $f_{\mathrm{ce}, \max }$ set to $900 \mathrm{~km}$ above the surface of Jupiter. Here an implicit assumption is that the peak loss of electrons on any magnetic field lines occurs at the same height as that of Jovian aurora ovals. Figure 3 shows the results, which look very similar to Figure 2. Some detailed parameters are listed in the last row of Table 1. Changing the assumed height from the planet's surface to $900 \mathrm{~km}$ above the surface can be seen to cause small differences in the model's results. Such minor differences are consistent with the parametric study of Louis et al. (2017). Though the influence of the height is small, we acknowledge that $900 \mathrm{~km}$ is a more realistic height for $f_{\mathrm{ce}}$ max.

\section{Statistical Results of Multiple DAMs Observed by Wind and STEREO Spacecraft}

To test the validity and reliability of our method, a statistical study is being carried out, of which preliminary results have been posted on arXiv (Zheng RB et al., 2022). In the ongoing study, we select 111 relatively strong and long-lasting DAM events observed by the WAVES instruments onboard at least two spacecraft of Wind (Bougeret et al., 1995), and STEREO-A and STEREO-B (Bougeret et al., 2008)] during 2008-September 2014. Among the 111 DAMs, 89 can be identified by type: A, B, C, or D (e.g., Ray and Hess, 2008), according to their central meridian longitude (CML)lo phase diagram (Marques et al., 2017) and the tilt of the Jovian magnetic dipole axis. Furthermore, the source regions (or active magnetic field lines) of 58 of the 89 DAMs can be successfully located by application of our method to radio observations from at least two spacecraft. Since the DAMs observed by Wind and STEREO are mostly from the southern hemisphere of Jupiter, to make 

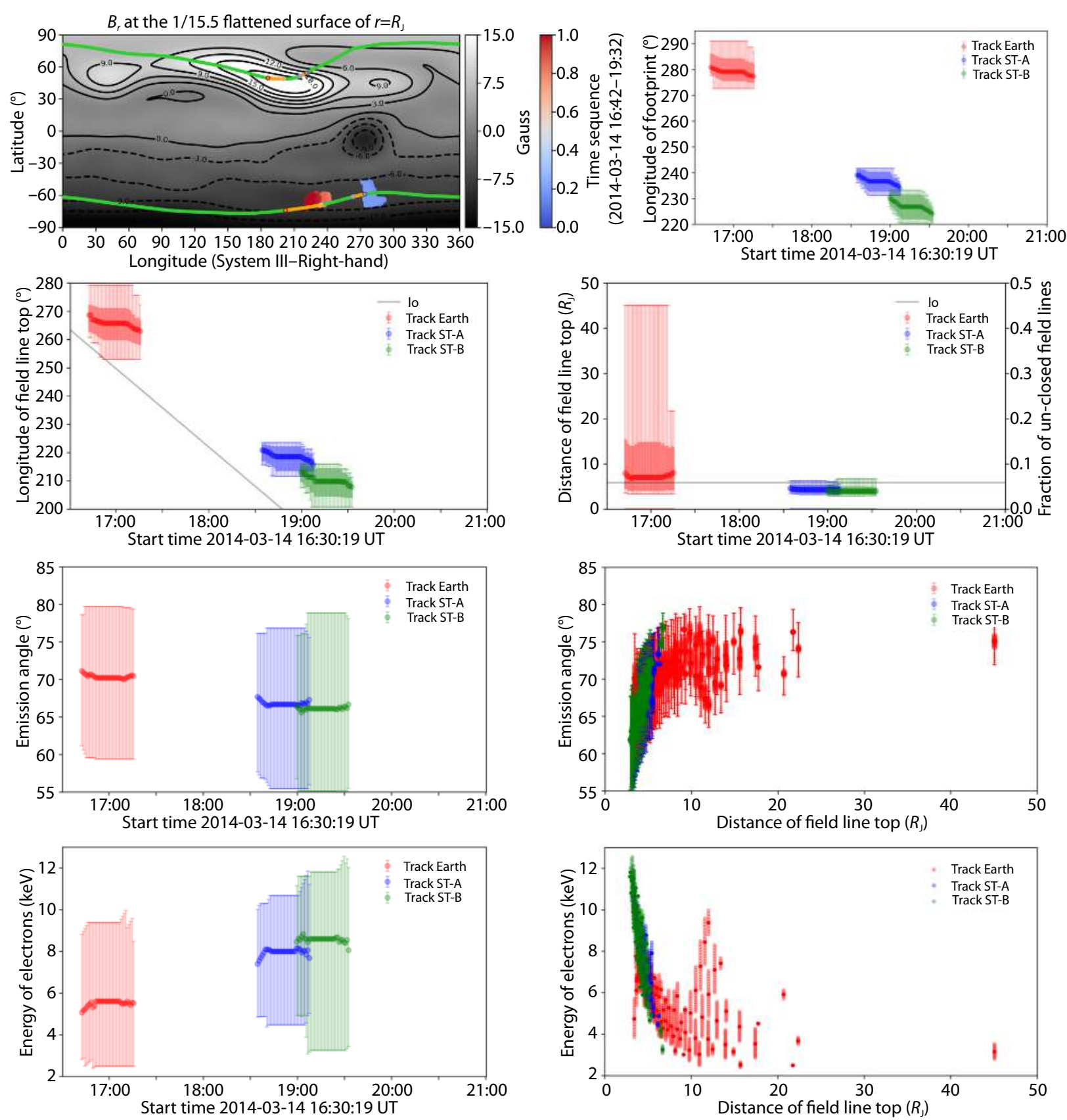

Figure 2. The updated Figs. 6-9 of Wang YM et al. (2020).

Table 1. Some characteristic parameters.

\begin{tabular}{|c|c|c|c|c|c|c|c|c|c|}
\hline$\Omega_{\mathrm{cc}}$ & $\Omega_{\mathrm{Io}}$ & $\Omega_{\mathrm{IFP}}$ & $\mathrm{LA}^{\mathrm{a}}$ & $\Omega_{\mathrm{fp}}$ & $\Omega_{\text {top }}$ & Distance $\left(R_{\mathrm{J}}\right)$ & $\mathrm{EA}^{\mathrm{b}}\left(^{\circ}\right)$ & Energy (keV) & Comments \\
\hline $0.255 \Omega$ & $0.23 \Omega$ ر & $0.55 \Omega$ & $32^{\circ}$ & $0.55 \Omega$ & $0.45 \Omega$ & $\sim\left(2.5_{-0.5}^{+1.5}-5.5_{-1.5}^{+2}\right)$ & $\sim\left(63.5_{-8.5}^{+9.5}-61.5_{-6.5}^{+10}\right)$ & $\sim\left(12.5_{-5}^{+3}-18_{-9}^{+6}\right)$ & W20 \\
\hline $0.255 \Omega$ ر & $0.23 \Omega$ & $0.37 \Omega$ & $\sim\left(7^{\circ}-20^{\circ}\right)$ & $0.37 \Omega$ & $0.33 \Omega$ & $\sim\left(8.4_{-3.8}^{+9.0}-4.1_{-0.8}^{+1.1}\right)$ & $\sim\left(71.5_{-10.2}^{+5.8}-67.8_{-11.2}^{+11.0}\right)$ & $\sim\left(4.6_{-2.3}^{+4.2}-7.2_{-3.9}^{+3.1}\right)$ & $900 \mathrm{~km}$ \\
\hline
\end{tabular}

Note: ${ }^{a}$ Lead angle of the footprints of the active field lines with respect to the Io footprint; ${ }^{b}$ Emission cone angle.

our sample more complete we have added another 10 northern

al., 1980; Lamy et al., 2017; 2021; Lecacheux, 2000).

DAMs observed by the Nancay Decameter Array (NDA, Boischot et 

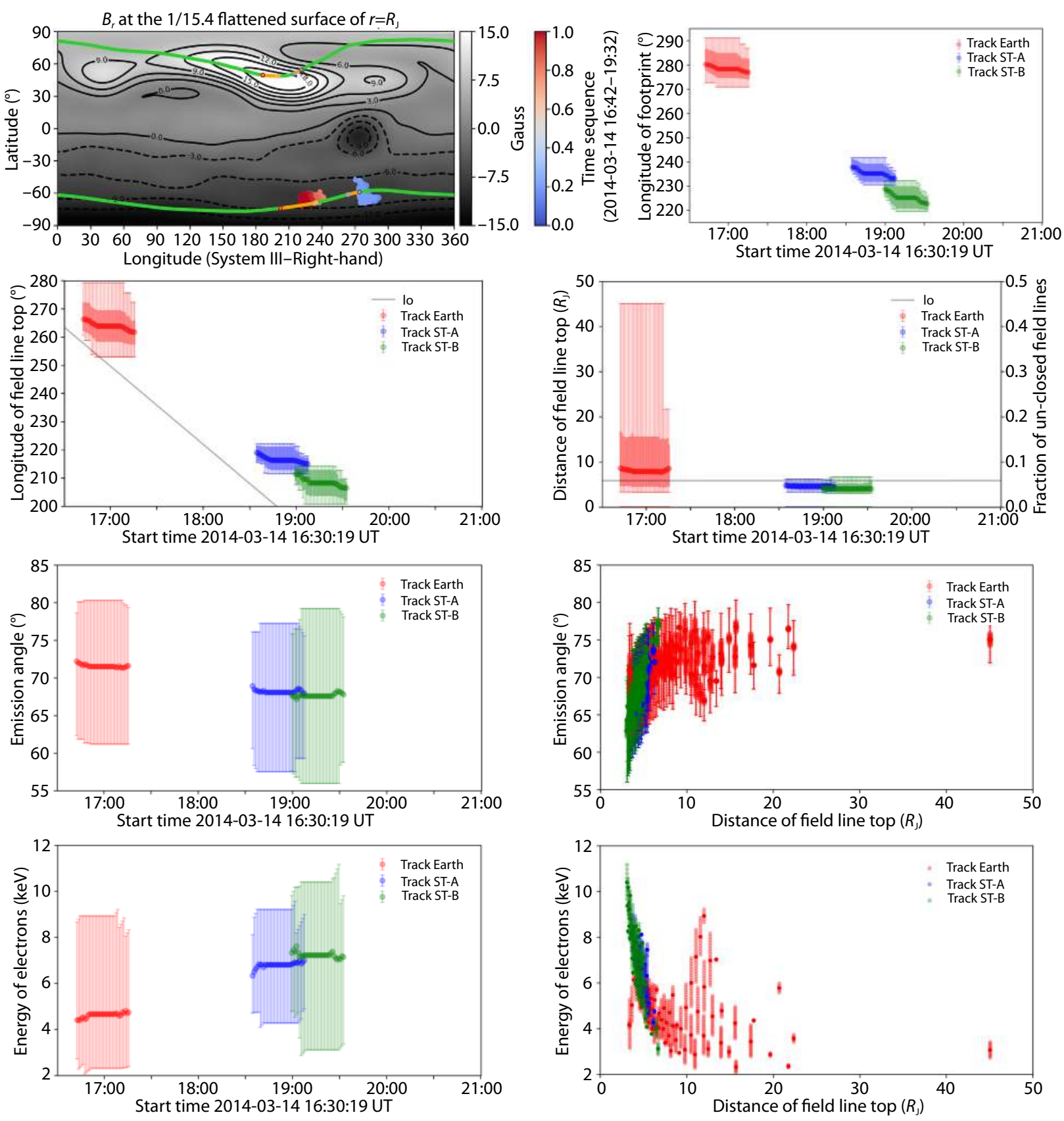

Figure 3. The same as Figure 2 except that $f_{\mathrm{ce}, \max }$ is set to the value of at $900 \mathrm{~km}$ above the surface of Jupiter instead of to the planet's surface.

We check the longitudes of the footprints (FPs) of the inferred active field lines of the 68 DAMs, which are shown in Figure 4. The results agree well with those of previous observational studies (Bonfond et al., 2017) and models (Hinton et al., 2019). In particular, the lead angle, defined as the longitude difference between the FPs and the footprint (IFP) of Io, varies between about $0^{\circ}$ and $25^{\circ}$, and the lead angles of the southern DAMs generally follow the modeled sinusoidal function of Hess et al. (2010) for the main Alfvén wing (MAW) auroral spots on the southern hemisphere (see Figure 4b). These consistencies suggest that our method is a promising tool to infer the source properties of DAMs as observed from remote spacecraft. More interesting parameters and detailed results of the statistical study of DAMs could be found in the paper by Zheng RB et al. (2022).

\section{Acknowledgments}

We gratefully acknowledge the use of data from the radio instruments on board Wind, and STEREO-A and B spacecraft. The authors thank the Nançay radio astronomy station/Nançay Scientific Unit of the Paris Observatory (USR 704-CNRS, supported by the University of Orleans, THE OSUC and the Centre Region in France) for providing access to NDA observations available online at https://www.obs-nancay.fr. The work done by the authors in China is supported by the Strategic Priority Program of the 

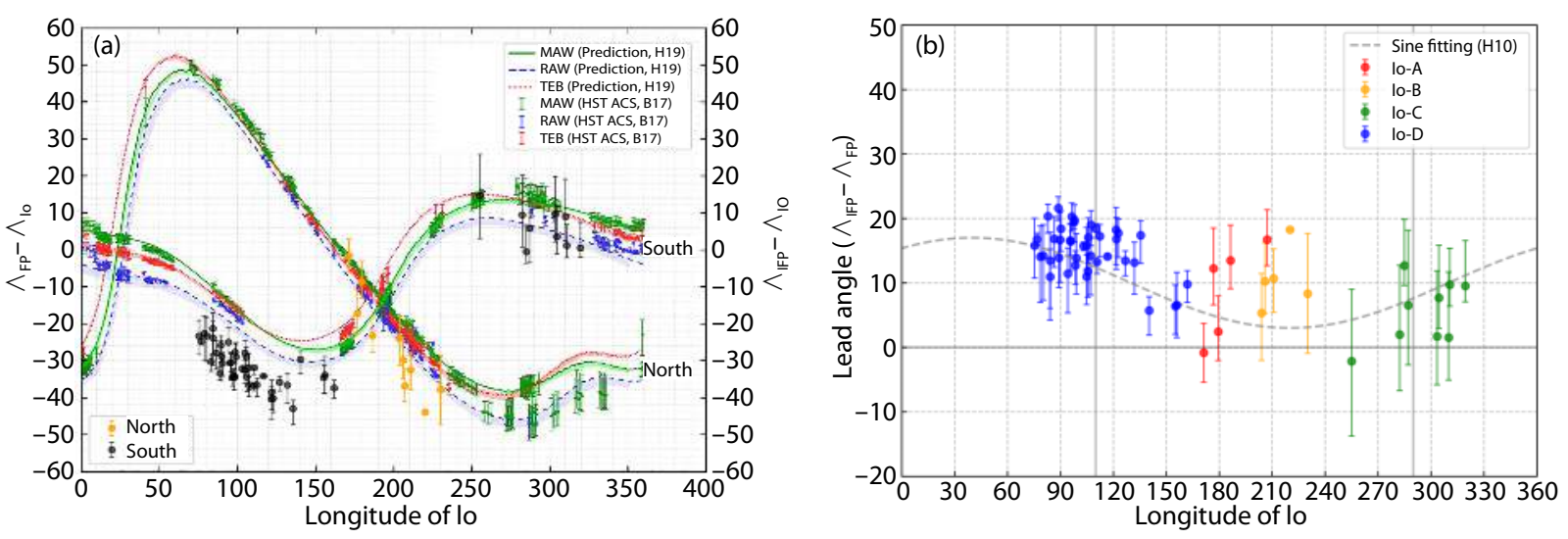

Figure 4. Panel (a) The longitude difference between the footprints (FPs) of active field lines and lo with orange color for the northern DAMs and black color for the southern DAMs. For comparison, the difference between the predicted (Hinton et al., 2019, or H19 in short) and observed (Bonfond et al., 2017, or B17 in short) longitudes of aurora spots, which are believed to be the footprint (IFP) of lo, and the longitdue of lo are also plotted, scaled by the $y$-axis on the right. Panel (b) The lead angle, defined as the longitude difference between the FP and IFP, of the DAMs. The modeled sinusoidal function by Hess et al. (2010, labeled as H10) for the main Alfvén wing (MAW) auroral spots in the southern hemisphere is also plotted for comparison. This figure is adapted from Zheng RB et al. (2022).

Chinese Academy of Sciences (Grant No. XDB41000000) and the NSFC (Grant Nos 42188101 and 42130204).

\section{References}

Boischot, A., Rosolen, C., Aubier, M. G., Daigne, G., Genova, F., Leblanc, Y., Lecacheux, A., De La Noë, J., and Møller-Pedersen, B. (1980). A new highgrain, broadband, steerable array to study Jovian decametric emission. Icarus, 43(3), 399-407. https://doi.org/10.1016/0019-1035(80)90185-2

Bonfond, B., Grodent, D., Gérard, J. C., Radioti, A., Dols, V., Delamere, P. A., and Clarke, J. T. (2009). The lo UV footprint: location, inter-spot distances and tail vertical extent. J. Geophys. Res. Space Phys., 114(A7), A07224. https://doi.org/10.1029/2009JA014312

Bonfond, B., Saur, J., Grodent, D., Badman, S. V., Bisikalo, D., Shematovich, V., Gérard, J. C., and Radioti, A. (2017). The tails of the satellite auroral footprints at Jupiter. J. Geophys. Res. Space Phys., 122(8), 7985-7996. https://doi.org/10.1002/2017JA024370

Bougeret, J. L., Kaiser, M. L., Kellogg, P. J., Manning, R., Goetz, K., Monson, S. J., Monge, N., Friel, L., Meetre, C. A., ... Hoang, S. (1995). WAVES: the radio and plasma wave investigation on the Wind spacecraft. Space Sci. Rev., 71(1-4), 231-263. https://doi.org/10.1007/BF00751331

Bougeret, J. L., Goetz, K., Kaiser, M. L., Bale, S. D., Kellogg, P. J., Maksimovic, M., Monge, N., Monson, S. J., Astier, P. L., ... Zouganelis, I. (2008). S/WAVES: the radio and plasma wave investigation on the STEREO mission. Space Sci. Rev., 136(1-4), 487-528. https://doi.org/10.1007/s11214-007-9298-8

Hess, S., Cecconi, B., and Zarka, P. (2008). Modeling of io-jupiter decameter arcs, emission beaming and energy source. Geophys. Res. Lett., 35(13), L13107. https://doi.org/10.1029/2008GL033656

Hess, S. L. G., Pétin, A., Zarka, P., Bonfond, B., and Cecconi, B. (2010). Lead angles and emitting electron energies of lo-controlled decameter radio arcs. Planet. Space Sci., 58(10), 1188-1198. https://doi.org/10.1016/j.pss.2010.04.011

Hinton, P. C., Bagenal, F., and Bonfond, B. (2019). Alfvén wave propagation in the lo plasma torus. Geophys. Res. Lett., 46(3), 1242-1249. https://doi.org/10.1029/2018GL081472

Lamy, L., Zarka, P., Cecconi, B., Klein, L., Masson, S., Denis, L., Coffre, A., and Viou, C. (2017). 1977-2017: 40 years of decametric observations of Jupiter and the Sun with the Nançay Decameter Array. Proceedings of the 8th International Workshop on Planetary, Solar and Heliospheric Radio Emissions held at Seggauberg near Graz, Austria, October 25-27, 2016, 43, 455-466. https://doi.org/10.1553/PRE8s455
Lamy, L., Cecconi, B., Aicardi, S., and Louis, C. K. (2020). Comment on "Locating the source field lines of Jovian decametric radio emissions" by YuMing Wang et al.. Earth Planet. Phys., 6(1), 10-12. https://doi.org/10.26464/epp2022018

Lamy, L., Le Gall, A., Cecconi, B., Loh, A., Renaud, P., Denis, L., Coffre, A., Zarka, P. and Lecacheux, A. (2021). Nançay Decameter Array (NDA) Jupiter Routine observation data collection (Version 1.7) [Data set]. PADC. https://doi.org/10.25935/DV2F-X016

Lecacheux, A. 2000. The Nançay Decameter Array: A Useful Step Towards Giant, New Generation Radio Telescopes for Long Wavelength Radio Astronomy. In Radio Astronomy at Long Wavelengths. In R. G. Stone, et al. (Eds.), AGU Geophys. Monogr. Ser., 119, 321.

Louarn, P., Allegrini, F., McComas, D. J., Valek, P. W., Kurth, W. S., André, N., Bagenal, F., Bolton, S., Connerney, J., ... Zink, J. L. (2017). Generation of the Jovian hectometric radiation: first lessons from Juno. Geophys. Res. Lett., 44(10), 4439-4446. https://doi.org/10.1002/2017GL072923

Louarn, P., Allegrini, F., McComas, D. J., Valek, P. W., Kurth, W. S., André, N., Bagenal, F., Bolton, S., Ebert, R. W., ...Wilson, R. J. (2018). Observation of electron conics by Juno: implications for radio generation and acceleration processes. Geophys. Res. Lett., 45(18), 9408-9416. https://doi.org/10.1029/2018GL078973

Louis, C. K. , Lamy, L. , Zarka, P. , Cecconi, B. , Hess, S. L. G. , and Bonnin, X. (2017). Simulating Jupiter-satellite decametric emissions with ExPRES: a parametric study. In Proceedings of the 8th International Workshop on Planetary Radio Emissions VIII (pp. 59-72). Vienna: Austrian Academy of Sciences Press. https://doi.org/10.1553/PRE8s59

Louis, C. K., Louarn, P., Allegrini, F., Kurth, W. S., and Szalay, J. R. (2020). Ganymede-induced decametric radio emission: in situ observations and measurements by Juno. Geophys. Res. Lett., 47(20), e2020GL090021. https://doi.org/10.1029/2020GL090021

Marques, M. S., Zarka, P., Echer, E., Ryabov, V. B., Alves, M. V., Denis, L., and Coffre, A. (2017). Statistical analysis of $26 \mathrm{yr}$ of observations of decametric radio emissions from Jupiter. Astron. Astrophys., 604(A17), 18.

Ray, L. C., and Hess, S. (2008). Modelling the lo-related DAM emission by modifying the beaming angle. J. Geophys. Res. Space Phys., 113(A11), A11218. https://doi.org/10.1029/2008JA013669

Wang, Y. M., Jia, X. Z., Wang, C. B., Wang, S., and Krupar, V. (2020). Locating the source field lines of Jovian decametric radio emissions. Earth Planet. Phys., 4(2), 95-104. https://doi.org/10.26464/epp2020015

Zheng, R. B. , Wang, Y. M. , Li, X. L. , Wang, C. B. and Jia, X. Z. (2022). Statistical study on the sources of Jovian decametric radio emissions based on the radio observations of remote instruments. arXiv: 2201.03148. 